\title{
Variação sazonal e diversidade de peixes do rio Cambará, Bacia do rio Uruguai
}

\author{
Carlos Eduardo Copatti ${ }^{1,2}$ \& Bethânia Ross Copatti ${ }^{1}$
}

\author{
${ }^{1}$ Curso de Ciências Biológicas, Centro de Ciências da Saúde, Universidade de Cruz Alta - Unicruz, \\ Rod. Municipal Jacob Della Méa, Km 5,6, Parada Benito, Campus Universitário Dr. Ulysses Guimarães, \\ CEP 98.020-290, Cruz, Alta, RS, Brasil \\ ${ }^{2}$ Autor para correspondência: Carlos Eduardo Copatti, e-mail: carloseduardocopatti@yahoo.com.br
}

COPATTI, C.E. \& COPATTI, B.R. Seasonal variation and diversity of fishes of the Cambará River, Uruguay Basin. Biota Neotrop. 11(4): http://www.biotaneotropica.org.br/v11n4/en/abstract?inventory+bn02611042011

\begin{abstract}
The aim of this study was to analyze the ictiofaun of Cambará River, Uruguay Basin, as well as the body dimensions of the organisms. The work was accomplished in two stretches of Cambará River ( $2^{\text {nd }}$ order) in Cruz Alta, RS. To the accomplishment of this research were performed identifications of individuals captured in May, September and November 2009 and February 2010 by using of gillnets, which remained in water for 24 hours, being checked every eight hours (1:00 and 9:00 AM and 5:00 P.M.). Besides the gillnets, were also used trawl (mesh $1.0 \mathrm{~mm}$ ), and fishing rods. The fish collected were cataloged and fixed in formaldehyde at $10.0 \%$ and then conserved in alcohol $70.0 \%$. In total, 420 individuals were identified, with a richness of 26 species and eight families. The species were classified in: constant (23.08\%), accessory (42.31\%) and accidental (34.61\%). Loricariidae (nine species and 156 individuals) and Characidae (seven species and 136 individuals) were the most typical. Among individuals collected are highlighted as more abundant: Hemiancistrus fuliginosus (89), Astyanax eigenmanniorum (50), and Rhamdia aff. quelen (44). One recorded species was of large mien (Hoplias lacerdae, with $164.92 \mathrm{~g}$ total weight and $23.40 \mathrm{~cm}$ total length), five medium sized species (98.55$164.92 \mathrm{~g}$ total weight and $19.56-21.50 \mathrm{~cm}$ total length) and 20 small species (less than $57.79 \mathrm{~g}$ total weight and $17.38 \mathrm{~cm}$ total length). The diversity was higher in hotter months, mainly between 1:00 and 9:00 A.M. The collector curve showed that still exist species of fish for being collected in Cambará River. Concluding that despite of the existence of environmental impacts in the stretch of study, it showed satisfactory diversity and richness, influenced by factors like heterogeneity of habitats and supply of allochthonouss material, as well as intrinsic factors (activity and reproduction) to the present species.
\end{abstract}

Keywords: Ictiofaunistic, Uruguay Basin, richness, constancy of occurrence.

COPATTI, C.E. \& COPATTI, B.R. Variação sazonal e diversidade de peixes do rio Cambará, Bacia do rio Uruguai. Biota Neotrop. 11(4): http://www.biotaneotropica.org.br/v11n4/pt/abstract?inventory+bn02611042011

Resumo: O objetivo deste estudo foi analisar a ictiofauna do rio Cambará, Bacia do rio Uruguai, bem como as dimensões corporais de seus representantes. O trabalho foi realizado em dois trechos do rio Cambará ( $2^{\mathrm{a}}$ ordem) em Cruz Alta/RS. Para a realização da pesquisa foram identificados exemplares capturados em maio, setembro e novembro de 2009 e fevereiro de 2010. Utilizaram-se redes de espera, que permaneceram expostas por 24 horas, sendo revisadas a cada oito horas (1; 9 e 17 horas). Além das malhas de espera, também se utilizou rede de arrasto (malha 1,0 mm) e varas de pesca. Os peixes coletados foram catalogados e fixados em formol a 10,0\% e depois conservados em álcool 70,0\%. Ao todo, identificaram-se 420 indivíduos, pertencentes a 26 espécies distribuídas em oito famílias. As espécies classificaram-se em: constantes (23,08\%), acessórias (42,31\%) e acidentais (34,61\%). As famílias Loricariidae (nove espécies e 156 indivíduos) e Characidae (sete espécies e 136 indivíduos) foram as mais abundantes. Entre os indivíduos coletados destacam-se como mais abundantes: Hemiancistrus fuliginosus (89), Astyanax eigenmanniorum (50) e Rhamdia quelen (44). Registraram-se uma espécie de grande porte (Hoplias lacerdae, com 164,92 g de peso total e 23,40 cm de comprimento total), cinco espécies de médio porte (98,55-164,92 $\mathrm{g}$ de peso total e 19,56-21,50 cm de comprimento total) e 20 espécies de pequeno porte (inferiores a $57,79 \mathrm{~g}$ de peso total e $17,38 \mathrm{~cm}$ de comprimento total). A diversidade coletada apresentou-se mais elevada nos meses mais quentes, principalmente entre 1 e 9 horas. A curva do coletor demonstrou que ainda existem espécies de peixes a serem coletadas no rio Cambará. Conclui-se que apesar da existência de impactos ambientais nos trechos de estudo, o mesmo demonstrou diversidade e riqueza satisfatórias, influenciados por fatores como heterogeneidade de habitats e oferta de material alóctone, bem como fatores intrínsecos (atividade e reprodução) das espécies presentes.

Palavras-chave: Ictiofauna, Bacia do rio Uruguai, riqueza, constância de ocorrência. 


\section{Introdução}

Nas últimas décadas tornou-se perceptível o aumento da população urbana, bem como dos processos industriais a ela atrelados. A preocupação decorre do fato de tais acontecimentos gerarem aumento da poluição no meio ambiente, especialmente em ambientes aquáticos. Segundo Copatti et al. (2009), fatores como ausência de mata ripária, despejos de efluentes domésticos e agrotóxicos lixiviados de lavouras próximas tendem a acelerar o estresse do ambiente. Além disso, alterações dos hábitats, tais como, canalização das margens, barragens, além de introduções de espécies não-nativas e a deterioração da qualidade da água, exercem uma profunda e negativa influência no ecossistema, podendo causar variações na distribuição espaço-temporal na comunidade de peixes (Waite \& Carpenter 2000). Trechos de rios cujo conteúdo longitudinal é artificialmente fragmentado, mas que são ricos em estruturas físicas podem suportar populações viáveis por fornecerem suficiente alimento e áreas de reprodução (Jungwirth et al. 2000).

Os ambientes aquáticos marinhos ou continentais abrigam grande diversidade de seres, incluindo algas, bactérias, macrófitas, artrópodes (crustáceos e insetos) e vertebrados (Nelson 1994). Dados recentes de Buckup et al. (2007) registram a ocorrência de 2.587 espécies de peixes de água doce distribuídos em 39 famílias no Brasil, o que demonstra a alta biodiversidade da região neotropical e afirma o aumento do conhecimento em relação à diversidade de peixes no Brasil.

As variações temporal e espacial das assembléias de peixes são influenciados por fatores bióticos e abióticos, os quais, de acordo com Jackson et al. (2001) e Okada et al. (2003) variam mesmo em ambientes próximos, uma vez que dependem das relações ecológicas entre as espécies, acessibilidade e disponibilidade de recursos e variações ambientais. Dessa forma, este trabalho teve como objetivo analisar a variação sazonal e a diversidade da ictiofauna do rio Cambará, Bacia do rio Uruguai, bem como as dimensões corporais de seus representantes.

\section{Material e Métodos}

\section{1. Área de estudo}

O estudo foi realizado em dois trechos do rio Cambará (rio de $2^{\mathrm{a}}$ ordem pertencente à Bacia do rio Uruguai) em Cruz Alta/RS, nas seguintes coordenadas: $28^{\circ} 30^{\prime} 55,21^{\prime}$ " $\mathrm{S}$ e $53^{\circ} 38^{\prime} 8,11^{\prime}$ " O (trecho 1 ) e $28^{\circ}{ }^{\circ} 33^{\prime} 29,60^{\prime}$ ' S e $53^{\circ} 36^{\prime} 33,60^{\prime \prime}$ O (trecho 2 ). Os trechos se distanciam entre si cerca de $7 \mathrm{~km}$. Ambos os trechos possuem águas turvas e as margens possuem mata ripária escassa e, inclusive, em muitos casos, inexistente.

No trecho 1 a profundidade varia de 0,5 a $3 \mathrm{~m}$ e a largura de 10 a $30 \mathrm{~m}$ com presença de ambientes lênticos, porém predominância de lóticos. O fundo apresenta áreas de pedregulhos, lages, sedimentos (lodo e/ou areia), bem como combinações destes substratos. No trecho 2 a profundidade varia de 0,5 a $1,8 \mathrm{~m}$ e a largura de 5 a $12 \mathrm{~m}$; possui predominância de ambientes lênticos, porém com regiões de lóticos. O fundo é composto principalmente por pedregulhos e algumas regiões com lages e sedimentos (lodo e/ou areia).

\section{Coleta}

A ictiofauna foi amostrada em maio, setembro e novembro de 2009 e fevereiro de 2010, através de uma coleta em cada estação do ano, perfazendo quatro coletas para cada trecho de estudo e oito coletas ao total.

Foram utilizadas redes de espera das seguintes malhas: $1,5 \mathrm{~cm}$ (uma rede de $5 \mathrm{~m}$ e outra de $10 \mathrm{~m}$ de comprimento); 2,0 $\mathrm{cm}$ (duas redes de $15 \mathrm{~m}$ de comprimento); 2,5 $\mathrm{cm}$ (uma rede de $15 \mathrm{~m}$ de comprimento); $3,0 \mathrm{~cm}$ (uma rede de $30 \mathrm{~m}$ de comprimento) e; $4,0 \mathrm{~cm}$ (uma rede de $30 \mathrm{~m}$ de comprimento). Todas as redes com $1 \mathrm{~m}$ de altura, exceto as redes de malhas 3,0 e 4,0 que possuem $2 \mathrm{~m}$ de altura. As redes foram colocadas em regiões diversas dos trechos de amostragem, de forma aleatória, abordando margem e centro. As redes de espera permaneceram na água por 24 horas, sendo revisadas a cada 8 horas $(9 ; 17$ e 1 hora). Cada período de revisão teve duração média de 50 minutos. Além das referidas redes, também foi utilizada rede de arrasto de malha $1,0 \mathrm{~mm}$ ( $5 \mathrm{~m}$ de comprimento e $0,5 \mathrm{~m}$ de altura). $\mathrm{O}$ arrasto foi realizado às 8 e às 16 horas e teve duração de 30 minutos. Por fim, foi procedida a utilização de varas de pesca sempre ao término de cada revisão das redes, com duração média de 90 minutos.

Os peixes coletados foram fixados em formol 10,0\%, onde permaneceram por 72 horas, sendo então transferidos para álcool $70,0 \%$ conforme Malabarba \& Reis (1987) e posteriormente identificados. De cada exemplar capturado registrou-se trecho de coleta, data, estação de amostragem, aparelho de pesca e período de captura. As medidas de peso e comprimento total e parcial foram realizadas em utilizando balança eletrônica com capacidade de $2.000 \mathrm{~g}$ e sensibilidade de $0,01 \mathrm{~g}$.

A identificação dos exemplares procedeu-se até o menor nível taxonômico possível utilizando-se bibliografia especializada para cada grupo taxonômico (Reis et al. 1990, Buckup \& Reis 1997, Zaniboni Filho et al. 2004, Rosso 2006, Buckup et al. 2007) e conhecimento pessoal.

\section{Análise da água}

Foram mensurados dados relativos de $\mathrm{pH}$, condutividade elétrica, alcalinidade total, oxigênio dissolvido, turbidez, amônia, cor e dureza total. Todas as análises foram feitas em Laboratório. Para cada trecho foi coletada uma amostra de água de 2,0 L para análise físico-química. As análises ocorreram de acordo com Clesceri \& Greenberg (2005). Os dados pluviométricos e de temperatura foram fornecidos pela Universidade de Cruz Alta-RS.

\section{Análise dos dados}

Para determinação das espécies residentes e espécies migrantes foi utilizada a constância de ocorrência, de fórmula: $\mathrm{C}=\mathrm{p} / \mathrm{P} \times 100$, onde $\mathrm{C}=$ valor de constância da espécie; $\mathrm{p}=$ número de coletas contendo a espécie estudada e; $\mathrm{P}=$ número total de coletas efetuadas. Neste estudo, uma espécie foi considerada constante quando C $>50 \%$, acessória, quando $25 \%<\mathrm{C} £ 50 \%$ e acidental quando $\mathrm{C} £ 25 \%$. Como espécies raras foram consideradas aquelas coletadas em apenas uma rede para um único trecho em uma única estação de coleta, porém independente do horário de registro.

A riqueza foi catalogada a cada estação do ano, em cada trecho de coleta, nos diferentes horários de captura. Para determinar se todas as espécies da ictiofauna existentes foram registradas, os dados foram tabulados para posterior elaboração da curva do coletor através do programa McAleece et al. (1997). O mesmo programa foi utilizado para calcular a diversidade de Shannon-Wienner (H'), a equitabilidade de Pielou (J'), a abundância e a dominância da espécie mais abundante em $\%(\mathrm{k})$.

\section{Resultados}

Ao longo das coletas, foram registrados 420 indivíduos, pertencentes a 26 espécies e oito famílias (Tabela 1). A análise demonstrou que as famílias Loricariidae (nove espécies e 156 indivíduos) e Characidae (sete espécies e 136 indivíduos) foram as mais representativas deste estudo em riqueza de espécies 
e número de indivíduos. Entre os indivíduos coletados, as espécies mais abundantes foram Hemiancistrus fuliginosus (89), Astyanmax eigenmanniorum (50) e Rhamdia quelen (44). Além disso, foi observada uma discrepância de riqueza entre fevereiro/2010, com 22 espécies, e setembro/2009 com dez espécies.

Apenas uma espécie (com um único indivíduo) de grande porto foi capturada: Hoplias lacerdae, com 164,92 g de peso total e 23,40 cm de comprimento total. Outras cinco espécies (Hoplias malabaricus, Hypostomus commersoni, Hypostomus isbrueckeri, R. quelen e Oligosarcus brevioris) com medidas entre 98,55 e 164,92 g de peso total e 19,56 e 21,50 cm de comprimento total foram consideradas de médio porte. As demais 20 espécies não ultrapassaram 57,79 g de peso total e $17,38 \mathrm{~cm}$ de comprimento total, sendo consideradas de pequeno porte. A maior ocorrência de espécies de pequeno porte está de acordo com o ambiente de coleta: rio de $2^{\mathrm{a}}$ ordem de pequenas dimensões.
Das 26 espécies registradas no rio Cambará, verificouse que apenas seis são constantes $(23,08 \%)$, ocorrendo ainda 11 acessórias $(42,31 \%)$ e nove acidentais $(34,61 \%)$, com oito raras $(30,77 \%)$ e uma não rara $(3,84 \%)$ (Tabela 2$)$.

Embora tenham sido observadas 26 espécies (Figura 1a), acreditase que muitas espécies acidentais (principalmente raras) não tenham sido coletadas, em virtude de sua menor distribuição, atividade, ocorrência e ausência de outros métodos de captura. Sendo assim analisou-se o estimador de riqueza Jack-knife1, o qual estimou em 33,88 espécies a riqueza no rio Cambará (Figura 1b), demonstrando a possibilidade de existir mais espécies a serem registradas.

A diversidade H' apresentou-se superior para o mês de fevereiro, tanto para o trecho $1(1,057)$, quanto para o trecho $2(0,979)$. A menor diversidade para o trecho 1 ocorreu em maio $(0,732)$, e para o trecho 2 em setembro (0,683). Em fevereiro ocorreu a maior equitabilidade J'

Tabela 1. Espécies de peixes do rio Cambará, Bacia do rio Uruguai, durante o período de 05/2009 a 02/2010.

Table 1. Species of fishes of the Cambará River, Uruguay Basin, during the period from 05/2009 to 02/2010.

\begin{tabular}{|c|c|c|c|c|c|c|c|c|c|c|}
\hline \multirow[t]{2}{*}{ Espécie } & \multicolumn{2}{|c|}{ Maio } & \multicolumn{2}{|c|}{ Set. } & \multicolumn{2}{|c|}{ Nov. } & \multicolumn{2}{|c|}{ Fev. } & \multirow[t]{2}{*}{ Método } & \multirow[t]{2}{*}{ Hora } \\
\hline & 1 & 2 & 1 & 2 & 1 & 2 & 1 & 2 & & \\
\hline \multicolumn{11}{|l|}{ ERYTHRINIDAE } \\
\hline Hoplias aff. malabaricus (Bloch, 1794) & 1 & 0 & 0 & 0 & 0 & 0 & 1 & 0 & 2,$0 ; 4,0$ & $a, b$ \\
\hline Hoplias aff. lacerdae (Miranda Ribeiro, 1908) & 0 & 0 & 0 & 0 & 0 & 0 & 1 & 0 & 2,5 & a \\
\hline \multicolumn{11}{|l|}{ HEPTAPTERIDAE } \\
\hline Rhamdia aff. quelen (Quoy \& Gaimard, 1824) & 2 & 6 & 5 & 0 & 5 & 4 & 14 & 8 & $\mathrm{VP} ; 1,0 ; 1,5 ; 2,0 ; 2,5 ; 3,0$ & $a, b, c$ \\
\hline Heptapterus mustelinus (Valenciennes, 1835) & 0 & 6 & 0 & 0 & 1 & 0 & 0 & 1 & $\mathrm{VP} ; 1,0$ & $a, b, c$ \\
\hline \multicolumn{11}{|l|}{ LORICARIIDAE } \\
\hline Loricariichthys anus (Valenciennes, 1835) & 1 & 0 & 0 & 0 & 0 & 0 & 0 & 0 & 1,5 & a \\
\hline Hemiancistrus fuliginosus (Cardoso \& Malabarba, 1999) & 0 & 56 & 3 & 4 & 1 & 7 & 1 & 17 & 1,$0 ; 1,5 ; 2,0 ; 2,5 ; 3,0 ; 4,0$ & $a, b, c$ \\
\hline Hypostomus commersoni (Valenciennes, 1836) & 1 & 0 & 3 & 1 & 3 & 2 & 4 & 4 & 2,$0 ; 2,5 ; 3,0 ; 4,0$ & $\mathrm{a}, \mathrm{c}$ \\
\hline Hypostomus isbrueckeri (Reis, Weber \& Malabarba, 1990) & 0 & 0 & 0 & 0 & 0 & 0 & 1 & 0 & 4,0 & a \\
\hline Hypostomus aspilogaster (Cope, 1894) & 0 & 12 & 0 & 0 & 0 & 18 & 0 & 9 & 1,$0 ; 1,5$ & $a, b$ \\
\hline Hypostomus ternetzi (Boulenger, 1895) & 0 & 1 & 0 & 0 & 0 & 0 & 0 & 0 & 2,0 & $\mathrm{c}$ \\
\hline Rineloricaria capitonia (Ghazzi, 2008) & 0 & 2 & 1 & 0 & 1 & 0 & 0 & 0 & 1,$0 ; 1,5 ; 2,0$ & $\mathrm{a}, \mathrm{c}$ \\
\hline Rineloricaria anitae (Ghazzi, 2008) & 0 & 0 & 0 & 0 & 0 & 2 & 0 & 0 & 1,$5 ; 3,0$ & a \\
\hline Ancistrus taunayi (Miranda Ribeiro, 1918) & 0 & 0 & 0 & 0 & 0 & 0 & 0 & 2 & 2,0 & a \\
\hline \multicolumn{11}{|l|}{ CRENUCHIDAE } \\
\hline Characidium pterostictum (Gomes, 1947) & 0 & 9 & 0 & 0 & 0 & 1 & 0 & 3 & 1,$0 ; 1,5$ & $a, b$ \\
\hline \multicolumn{11}{|l|}{ TRICOMYCTERIDAE } \\
\hline Trichomycterus sp. & 0 & 10 & 0 & 1 & 0 & 0 & 0 & 4 & 1,0 & $a, b$ \\
\hline \multicolumn{11}{|l|}{ CICHLIDAE } \\
\hline Crenicichla missioneira (Lucena \& Kullander, 1992) & 0 & 0 & 0 & 0 & 2 & 0 & 6 & 1 & $\mathrm{VP} ; 2,0$ & $\mathrm{a}, \mathrm{c}$ \\
\hline Crenicichla sp. & 0 & 0 & 0 & 0 & 0 & 0 & 1 & 0 & 2,0 & $\mathrm{~b}$ \\
\hline Gymnogeophagus aff. gymnogenys (Hensel, 1870) & 0 & 0 & 0 & 0 & 22 & 0 & 11 & 0 & $\mathrm{VP} ; 2,0$ & $\mathrm{a}, \mathrm{b}, \mathrm{c}$ \\
\hline \multicolumn{11}{|l|}{ ANASTOMIDAE } \\
\hline Schizodon aff. nasutus (Kner, 1858) & 0 & 0 & 0 & 0 & 0 & 0 & 1 & 0 & 1,5 & a \\
\hline \multicolumn{11}{|l|}{ CHARACIDAE } \\
\hline Oligosarcus brevioris (Menezes, 1987) & 0 & 0 & 1 & 0 & 1 & 0 & 5 & 5 & VP; 1,$5 ; 2,0 ; 2,5 ; 3,0$ & $a, b, c$ \\
\hline Oligosarcus jenynsii (Günther, 1864) & 2 & 1 & 0 & 0 & 0 & 0 & 1 & 1 & 1,$5 ; 2,0$ & $a, b, c$ \\
\hline Galeocharax humeralis (Valenciennes, 1834) & 0 & 0 & 0 & 0 & 0 & 0 & 1 & 0 & 2,0 & $\mathrm{c}$ \\
\hline Bryconamericus iheringii (Boulenger, 1887) & 0 & 0 & 3 & 5 & 9 & 1 & 3 & 0 & VP; 1,5 & $a, b, c$ \\
\hline Astyanax jacuhiensis (Cope, 1894) & 2 & 0 & 0 & 1 & 1 & 0 & 3 & 0 & VP; 1,5 & $a, b$ \\
\hline Astyanax eigenmanniorum (Cope, 1894) & 9 & 2 & 1 & 6 & 10 & 6 & 7 & 9 & VP; 1,$0 ; 1,5 ; 2,0 ; 3,0$ & $a, b, c$ \\
\hline Astyanax aff. fasciatus (Cuvier, 1819) & 1 & 3 & 0 & 13 & 7 & 6 & 6 & 4 & VP; 1,$5 ; 2,0$ & $a, b, c$ \\
\hline
\end{tabular}

Trecho $1=1$; Trecho $2=2$. Vara de pesca $=$ VP. Horário: $1-9$ horas $=\mathrm{a} ; 9-17$ horas $=\mathrm{b} ; 17-1$ horas $=\mathrm{c}$.

Stretch $1=1 ;$ Stretch $2=2$. Fishing rod = VP. Horary: 1:00-9:00 AM = a; 9:00 AM-5:00 PM = b; 5:00 PM-1:00 AM =c. 
Copatti, C.E. \& Copatti, B.R.

Tabela 2. Média de Peso, Comprimento Total (CT) e Comprimento Parcial (CP) dos peixes do rio Cambará, Bacia do rio Uruguai, durante o período de 05/2009 a 02/2010.

Table 2. Weight, Total Length (CT) and Partial Length (CP) of the fishes of the Cambará River, Uruguay Basin, during the period from 05/2009 to 02/2010.

\begin{tabular}{|c|c|c|c|c|}
\hline Espécie & Constância & Peso (g) & $\mathrm{CT}(\mathrm{cm})$ & $\mathbf{C P}(\mathrm{cm})$ \\
\hline \multicolumn{5}{|l|}{ ERYTHRINIDAE } \\
\hline Hoplias aff. malabaricus & $\mathrm{B}$ & 118,62 & 21,50 & 17,70 \\
\hline Hoplias aff. lacerdae & $\mathrm{CR}$ & 164,92 & 23,40 & 20,00 \\
\hline \multicolumn{5}{|l|}{ HEPTAPTERIDAE } \\
\hline Rhamdia aff. quelen & A & 98,72 & 20,53 & 17,44 \\
\hline Heptapterus mustelinus & $\mathrm{B}$ & 17,40 & 12,50 & 11,22 \\
\hline \multicolumn{5}{|l|}{ LORICARIIDAE } \\
\hline Loricariichthys anus & $\mathrm{CR}$ & 19,60 & 14,80 & 13,00 \\
\hline Hemiancistrus fuliginosus & A & 56,18 & 16,21 & 12,71 \\
\hline Hypostomus commersoni & A & 117,81 & 22,84 & 17,20 \\
\hline Hypostomus isbrueckeri & $\mathrm{CR}$ & 114,70 & 23,00 & 18,00 \\
\hline Hypostomus aspilogaster & B & 3,57 & 6,61 & 5,39 \\
\hline Hypostomus ternetzi & $\mathrm{CR}$ & 28,33 & 13,90 & 10,40 \\
\hline Rineloricaria capitonia & B & 8,44 & 9,22 & 8,02 \\
\hline Rineloricaria anitae & $\mathrm{C}$ & 14,80 & 11,15 & 9,35 \\
\hline Ancistrus taunayi & $\mathrm{CR}$ & 21,01 & 11,10 & 9,20 \\
\hline \multicolumn{5}{|l|}{ CRENUCHIDAE } \\
\hline Characidium pterostictum & $\mathrm{B}$ & 3,18 & 6,48 & 5,46 \\
\hline \multicolumn{5}{|l|}{ TRICOMYCTERIDAE } \\
\hline Trichomycterus sp. & $\mathrm{B}$ & 0,82 & 4,68 & 4,11 \\
\hline \multicolumn{5}{|l|}{ CICHLIDAE } \\
\hline Crenicichla missioneira & $\mathrm{B}$ & 36,93 & 14,84 & 12,06 \\
\hline Crenicichla sp. & $\mathrm{CR}$ & - & - & - \\
\hline Gymnogeophagus aff. gymnogenys & B & 37,80 & 12,41 & 9,73 \\
\hline \multicolumn{5}{|l|}{ ANASTOMIDAE } \\
\hline Schizodon aff. nasutus & $\mathrm{CR}$ & 19,45 & 11,50 & 9,30 \\
\hline \multicolumn{5}{|l|}{ CHARACIDAE } \\
\hline Oligosarcus brevioris & $\mathrm{B}$ & 98,55 & 19,56 & 16,48 \\
\hline Oligosarcus jenynsii & $\mathrm{B}$ & 57,79 & 17,38 & 14,44 \\
\hline Galeocharax humeralis & $\mathrm{CR}$ & 49,07 & 16,00 & 13,30 \\
\hline Bryconamericusiheringii & A & 7,56 & 7,95 & 6,53 \\
\hline Astyanax jacuhiensis & $\mathrm{B}$ & 8,46 & 8,48 & 6,78 \\
\hline Astyanax eigenmanniorum & A & 28,20 & 11,97 & 9,77 \\
\hline Astyanax aff. fasciatus & A & 24,03 & 11,64 & 9,49 \\
\hline
\end{tabular}

Constância de ocorrência: Constante $=\mathrm{A} ;$ Acessória $=\mathrm{B} ;$ Acidental $=\mathrm{C} ;$ Rara $=\mathrm{R}$.

Constancy of occurrence: Constant $=\mathrm{A}$; Accessory = B; Accidental $=\mathrm{C}$; Rare $=\mathrm{R}$.

e a menor dominância para o rio Cambará. Além disso, nesse mesmo mês ocorreu a maior riqueza de espécies para ambos os trechos, bem como a maior abundância (Tabela 3).

Neste estudo os peixes com atividade entre 1 e 9 horas apresentaram a maior diversidade $\mathrm{H}^{\prime}$, tanto para o trecho $1(1,087)$, quanto para o trecho 2 (1,011). A equitabilidade $\mathrm{J}$ ' foi maior no trecho $1(0,917)$ no período das 9 às 17 horas e para o trecho $2(0,860)$, entre 1 e 9 horas. Ainda na interface madrugada/amanhecer, ocorreu a maior riqueza e a menor dominância (Tabela 4). Entre os horários de coleta, maior abundância e riqueza ocorreram entre as 17-1 horas (197 indivíduos e 15 espécies), seguido de 1-9 horas (140 indivíduos e 23 espécies) e 9-17 horas (82 indivíduos e 15 espécies) (Tabela 4).

Também foram avaliados os parâmetros físico-químicos da água (Tabela 5), que se demonstraram dentro da normalidade, não comprometendo a qualidade dos ambientes amostrados. Os índices pluviométricos e as médias de temperatura para maio, setembro e novembro/2009 e fevereiro/2010 foram, respectivamente: $165 ; 408$; 500 e $143 \mathrm{~mm}$ e 20,$5 ; 17,0 ; 22,0$ e $25,0{ }^{\circ} \mathrm{C}$.

\section{Discussão}

Diversos estudos já foram efetuados na Bacia do rio Uruguai para o Rio Grande do Sul. Weis et al. (1983) no levantamento da fauna ictiológica do rio Ibicuí-Mirim, constataram a ocorrência de 81 espécies. Bossemeyer et al. (1985) coletaram um total de 1.345 peixes pertencentes a 53 espécies no rio Santa Maria. Bertoletti et al. (1989a) estudando a ictiofauna do rio Uruguai em seu trecho superior (entre Aratiba e Esmeralda-RS) encontraram 74 espécies, sendo que oito delas foram consideradas restritas à região. Em trabalho desenvolvido no mesmo ano sobre a ictiofauna do rio Canoas, Bertoletti et al. 
Tabela 3. Diversidade de Shannon Log Base 10 (H'); Equitabilidade de Pielou (J'); Riqueza Total (S); Número de Indivíduos (n) e; Dominância (k) de peixes do rio Cambará, Bacia do rio Uruguai, durante o período de 05/2009 a $02 / 2010$.

Table 3. Shannon Log Base 10 Diversity (H'); Pielou Evenness (J'); Total Richness (S); Number of Individuals (n) e; Dominance (k) of fishes of the Cambará River, Uruguay Basin during the period from 05/2009 to 02/2010.

\begin{tabular}{lcccccccc}
\hline Índice & \multicolumn{2}{c}{ Maio } & \multicolumn{2}{c}{ Set. } & \multicolumn{2}{c}{ Nov. } & \multicolumn{2}{c}{ Fev. } \\
\cline { 2 - 9 } & $\mathbf{1}$ & $\mathbf{2}$ & $\mathbf{1}$ & $\mathbf{2}$ & $\mathbf{1}$ & $\mathbf{2}$ & $\mathbf{1}$ & $\mathbf{2}$ \\
\hline H' & 0,732 & 0,724 & 0,772 & 0,683 & 0,854 & 0,790 & 1,057 & 0,979 \\
J' & 0,810 & 0,695 & 0,914 & 0,808 & 0,791 & 0,828 & 0,859 & 0,878 \\
S & 8 & 11 & 7 & 7 & 12 & 9 & 17 & 13 \\
n & 19 & 108 & 17 & 31 & 63 & 47 & 67 & 68 \\
k (\%) & 47,37 & 51,85 & 29,41 & 41,94 & 34,92 & 38,30 & 20,90 & 25,00 \\
\hline
\end{tabular}

Trecho $1=1 ;$ Trecho $2=2$.

Stretch $1=1 ;$ Stretch $2=2$.

Tabela 4. Diversidade de Shannon Log Base $10\left(\mathrm{H}^{\prime}\right)$; Equitabilidade de Pielou (J'); Riqueza Total (S); Número de Indivíduos (n) e; Dominância (k) de peixes do rio Cambará, Bacia do rio Uruguai, para os diferentes horários de coleta.

Table 4. Shannon Log Base 10 Diversity (H'); Pielou Evenness (J'); Total Richness (S); Number of Individuals (n) e; Dominance (k) of fishes of the Cambará River, Uruguay Basin for the different sampling times.

\begin{tabular}{lcccccc}
\hline Índice & \multicolumn{2}{c}{$\mathbf{1 - 9}$ horas } & \multicolumn{2}{c}{$\mathbf{9 - 1 7}$ horas } & \multicolumn{2}{c}{$\mathbf{1 7 - 1}$ hora } \\
\cline { 2 - 7 } & $\mathbf{1}$ & $\mathbf{2}$ & $\mathbf{1}$ & $\mathbf{2}$ & $\mathbf{1}$ & $\mathbf{2}$ \\
\hline H' & 1,087 & 1,011 & 0,875 & 0,777 & 0,885 & 0,605 \\
J' & 0,903 & 0,860 & 0,917 & 0,814 & 0,820 & 0,605 \\
S & 16 & 15 & 09 & 09 & 12 & 10 \\
n & 47 & 93 & 24 & 58 & 95 & 102 \\
k (\%) & 14,89 & 23,66 & 25,00 & 34,48 & 27,37 & 60,78 \\
\hline
\end{tabular}

Trecho $1=1 ;$ Trecho $2=2$.

Stretch $1=1 ;$ Stretch $2=2$.

Tabela 5. Valores médios ( \pm erro padrão) dos parâmetros físico-químicos da água do rio Cambará, Bacia do rio Uruguai.

Table 5. Mean ( \pm standard error) of the physic-chemical parameters of water of the Cambará River, Uruguay Basin.

\begin{tabular}{llr}
\hline \multicolumn{1}{c}{ Parâmetros } & \multicolumn{1}{c}{ VMP } & \multicolumn{1}{c}{ Resultado } \\
\hline $\mathrm{O}_{2}$ dissolvido & $>5 \mathrm{mg} \cdot \mathrm{L}^{-1}$ & $11,34 \pm 0,86$ \\
$\mathrm{pH}$ & $6,0-9,0$ & $6,62 \pm 0,91$ \\
Amônia $\left(\mathrm{mg} . \mathrm{L}^{-1} \mathrm{NH}_{3}\right)$ & $<1,5$ & $0,20 \pm 0,12$ \\
Alcalinidade total $\left(\mathrm{mg}_{\mathrm{L}} \mathrm{L}^{-1} \mathrm{CaCO}_{3}\right)$ & $\mathrm{NC}$ & $60 \pm 5,40$ \\
Dureza total $\left(\mathrm{mg} . \mathrm{L}^{-1} \mathrm{CaCO}_{3}\right)$ & $\mathrm{NC}$ & $20 \pm 4,08$ \\
Turbidez & $<100 \mathrm{NTU}$ & $7,56 \pm 1,07$ \\
Cor & $<75 \mathrm{mg} . \mathrm{L}^{-1} \mathrm{Pt} . \mathrm{L}^{-1}$ & $17,92 \pm 3,89$ \\
Condutividade $\left(\mathrm{mS} . \mathrm{cm}^{-1} \mathrm{a} 25^{\circ} \mathrm{C}\right)$ & $\mathrm{NC}$ & $25,9 \pm 4,87$ \\
\hline
\end{tabular}

$\mathrm{VMP}=$ valores médios permitidos.

$\mathrm{VMP}=$ average allowed.

(1989b) verificaram a existência de 53 espécies. Bertoletti et al. (1990) capturaram 5.638 exemplares de peixes pertencentes a 71 espécies na usina hidrelétrica de Garabi no rio Uruguai e Malabarba \& Isaia (1992) registraram 73 espécies para o sistema do rio Tramandaí, sendo que destas, 33 são citadas pela primeira vez para a referida área. Mais recentemente, Behr (2005) registrou a ocorrência de 111 espécies distribuídas em 29 famílias para o rio Ibicuí.

Trabalhos realizados em rio de menor porte desta Bacia também se destacam. Azevedo et al. (2003) estudando o arroio Felizardo
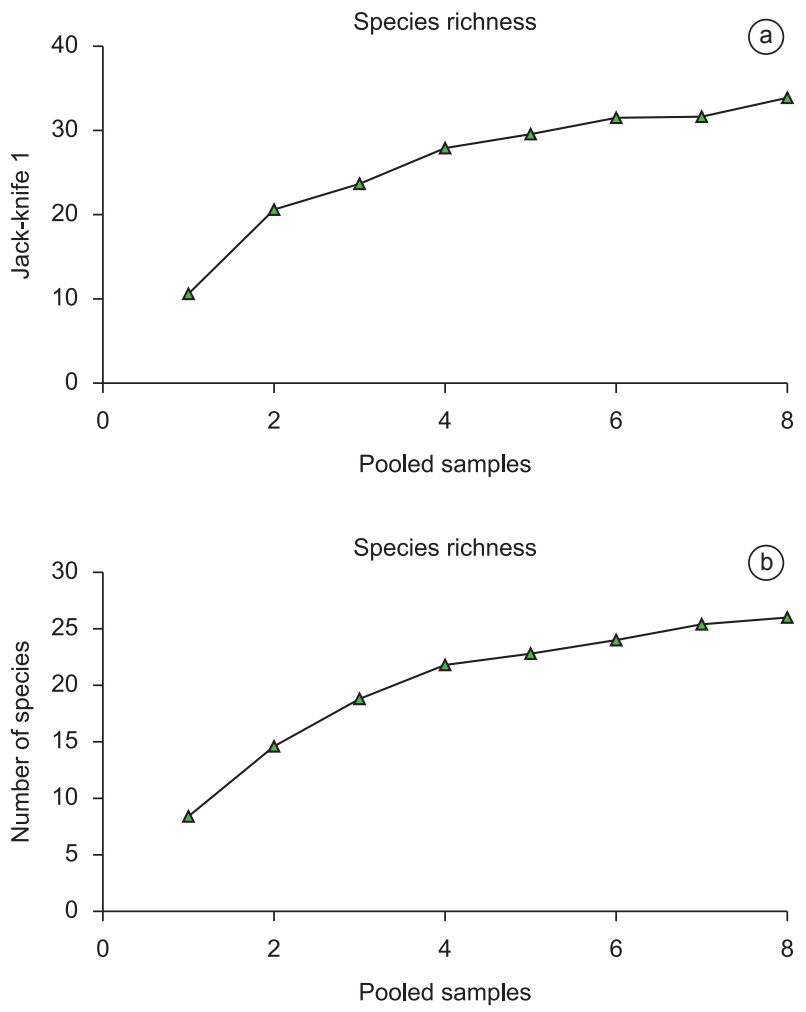

Figura 1. Curva do coletor (riqueza de espécies $\times$ sequência de amostras) para as: a) espécies observadas; e b) estimadas de acordo com Jack-knife1 do rio Cambará, Bacia do rio Uruguai, durante o período de 05/2009 a 02/2010.

Figure 1. Collector curve (species richness $\times$ sequence of samples) to the: a) species observed; and b) estimated using Jack-knife 1 of the Cambará River, Uruguay Basin, during the period from 05/2009 to 02/2010.

(Uruguaiana-RS), registraram 27 espécies e 11 famílias pelo método de captura de pesca elétrica. Pessano et al. (2005) encontraram 37 espécies, 27 gêneros e 14 famílias no arroio Quarai-Chico, afluente do rio Uruguai-RS, situado em área de conservação ambiental. Copatti et al. (2009) identificaram um total de 572 indivíduos no rio Jaguari, distribuídos em 14 famílias e 26 espécies. Kaufmann \& Pinheiro (2009), no Arroio Fortaleza, coletaram 527 exemplares de peixes, de 25 espécies, 13 gêneros e sete famílias. Para o rio Cambará registrou-se 26 espécies, dado similar a trabalhos realizados em rios de porte similar, mesmo apresentando extensão e vazão inferior a maioria dos rios citados.

Segundo Copatti et al. (2009), uma variação na riqueza de espécies e até mesmo no número de indivíduos pode ser esperada quando se considera a sazonalidade. No presente trabalho isto se verificou, pois além da riqueza, a abundância também sofreu mudanças perceptíveis, com 135 e 48 indivíduos em fevereiro/2010 e setembro/2009, respectivamente. Já maio e novembro/2009 apresentaram respectivamente 127 e 110 indivíduos (com riqueza de 15 espécies em ambos os meses).

A variação da riqueza demonstra que as comunidades são afetadas pelas mudanças sazonais que implicam na alteração dos parâmetros abióticos, que interferem diretamente na dinâmica populacional das espécies. Para Wootton (1990), as redes de espera, por serem artes de pesca passivas, sofrem influência da movimentação dos peixes, que é mais ativa em períodos em que a temperatura é mais elevada. Neste estudo, setembro/2009 apresentou as médias mais baixas de temperatura, bem como menor riqueza e abundância de espécies. Oliveira \& Garavello (2003) citam que a constância pode ser influenciada pelos equipamentos de pesca utilizados nas coletas, 
já que eles apresentam certa seletividade qualitativa de espécies e de tamanhos de indivíduos. Dessa forma, o uso de varas de pesca pode ter contribuído para aumentar a riqueza encontrada.

Em riachos tropicais, o aumento da diversidade ao longo do rio tem sido associado por vários autores a um aumento na heterogeneidade de habitats, o qual, por sua vez, é relacionado principalmente a um aumento na vazão e na disponibilidade de abrigos (Viana 1989, Peres-Neto et al. 1995, Castro et al. 2003a, b). Para este estudo, aparentemente existe mudanças na estrutura da ictiofauna ao longo do rio, uma vez que oito espécies foram exclusivas para o trecho 1 e seis para o trecho 2 , demonstrando que mesmo ambientes próximos podem apresentar espécies exclusivas, porém apenas com um esforço amostral maior (incluído novos métodos de coleta) será possível confirmar tal informação. Contudo, os resultados de Viana (1989) dão suporte a este dado, pois a caracterização da estruturação da taxocenose íctica no Ribeirão Santana (Bacia do alto rio Paraná, DF), verificou que em resposta ao aumento da estabilidade e complexidade do ambiente, ao longo do gradiente longitudinal, há um aumento na diversidade de espécies, biomassa e guildas tróficas.

Nos grandes rios sul-americanos, com grandes planícies de inundação, a abundância dos peixes detritívoros está, provavelmente, associada à abundância de material detrítico e ao êxito no seu aproveitamento (Yossa-Perdomo 2002). As famílias Loricariidae e Characidae foram as mais representativas deste estudo (Tabela 1). Ambas possuem vários representantes de hábito detritívoro, o que demonstra uma grande necessidade de material alóctone (geralmente provenientes de restos vegetais da mata ripária) para fornecer a carga energética necessária ao estabelecimento de uma rede alimentar de detritos, intrinsecamente correlacionada a uma rede alimentar herbívora, as quais estruturam e mantém redes tróficas em homeostase. Dessa forma, a manutenção da vegetação ripariana é condição essencial para manter uma comunidade ictiofaunística em constante desenvolvimento, especialmente daqueles exemplares com baixa capacidade de deslocamento, dependentes essencialmente das condições locais existentes. Como a mata ripária nos trechos de pesquisa se encontra deveras fragmentada, isto pode indicar um alto risco de extinção local, não só para espécies diretamente dependentes destes detritos, mas também a todas as demais correlacionadas a estas.

De acordo com Pianka (1974), espécies de pequeno porte são estrategistas $\mathrm{k}$, pois a alocação de recursos alimentares e o espaço físico são limitados para rios de menor porte. Além disso, as espécies exibem adaptações compatíveis ao hábitat (pequeno porte), como: morfologia do corpo através de maior desenvolvimento das nadadeiras peitorais (Crenuchidae), placas ósseas revestindo o corpo (Loricariidae) e espinhos ósseos operculares (Trichomycteridae); hábitos alimentares bentófagos (em especial larvas e ninfas de insetos) e; baixa fecundidade (ovócitos grandes e em pequeno número, redução de gônadas) (Braga, 2004).

Casatti et al. (2001) contabilizaram que aproximadamente $73 \%$ das espécies e $96 \%$ dos indivíduos de peixes de uma Bacia do alto rio Paraná são de pequeno porte, reforçando a opinião de Casatti \& Castro (1998) que consideram a predominância de peixes de pequeno porte como o único padrão geral com valor diagnóstico para a ictiofauna de riachos sul-americanos. Ainda segundo os mesmos autores, associados ao pequeno porte destes peixes, seu grau relativamente elevado de endemismo e sua ocupação de microhabitat bastante específicos são fatores que acentuam ainda mais a necessidade de estabelecer estratégias para a conservação desses ambientes. DalaCorte et al. (2009), em estudo na Floresta Nacional de Canela, Bacia do rio Caí, em três rios de $2^{\mathrm{a}}$ ordem (Caçador, Saiqui e Tiririca) capturaram espécies de pequeno porte, destacando entre elas Trichomycterus sp., uma espécie ainda não descrita.
Apone et al. (2008) no estudo da ictiofauna do rio Quilombo observaram uma diminuição das espécies classificadas como constantes $(65,08 \%)$ e um aumento de espécies acessórias $(14,26 \%)$ e acidentais $(20,66 \%)$ a partir das cabeceiras do rio Quilombo em direção a sua foz. Neste estudo, as espécies constantes $(23,08 \%)$ ocorreram em menor número relativo às acessórias $(42,31 \%)$ e acidentais $(34,61 \%)$. Fatores como a diminuição da altitude, aumento do volume de água, da largura e da profundidade e maior diversidade de micro-habitats podem proporcionar ambientes mais facilmente colonizáveis do que aqueles encontrados nas cabeceiras. Shibatta et al. (2007), em estudo do rio Tibagi, capturaram 2.758 indivíduos nos quatro trechos de coleta, entre eles destacamse espécies classificadas como constantes $(25,16 \%)$, um maior índice de espécies acessórias $(39,79 \%)$ e acidentais $(29,16 \%)$, representando quatro espécies raras $(5,88 \%)$. Kaufmann \& Pinheiro (2009) em trabalho no Arroio Fortaleza, afluente do rio Uruguai, obtiveram diversidade H' que variou entre 1,43 e 2,68 entre os seis trechos. Estes valores representam grande diversidade de espécies. A equitabilidade $\mathrm{J}$ ' foi sempre superior a 0,80 , indicando que os indivíduos estão bem distribuídos entre as diferentes espécies.

Lowe-McConnell (1975) menciona que o período do dia é outro fator determinante da taxa de alimentação das espécies, havendo aquelas que se alimentam preferencialmente durante o período diurno, enquanto outras apresentam hábitos alimentares noturnos. De modo geral, neste trabalho, percebe-se que o pico das atividades ocorreu no horário noturno, especialmente na interface madrugada/amanhecer. Copatti et al. (2009) em estudo no rio Jaguari, relataram que a maior abundância ocorreu entre 18-24 horas, o que demonstra a preferência dos peixes em realizar suas atividades de captura de alimento e deslocamento, entre outras, no período noturno. Saccol-Pereira \& Fialho (2010) verificaram que a distribuição da assembléia de peixes no Delta do Jacuí foi influenciada principalmente pela temperatura da água. A estrutura da assembléia apresentou maior abundância no outono, porém, na comparação entre dia e noite, não se averiguou diferenças para abundância.

Apesar do rio Cambará apresentar impactos ambientais, destacandose mata ripária reduzida e entrada de resíduos de agrotóxicos provindos de lavouras circunvizinhas, bem como ser um rio de pequeno porte ( $2^{\mathrm{a}}$ ordem), o mesmo demonstrou diversidade e riqueza satisfatórias, inclusive com muitas espécies raras. Os principais fatores que contribuíram para elevar a diversidade foram: heterogeneidade de habitats, representada por trechos lênticos e lóticos e tipo de substrato (pedregoso, lodoso e arenoso) e; oferta de material alóctone (folhas e demais partes vegetais que se destacam da vegetação ripariana ou são arrastados pela lixiviação). Além disso, fatores externos como temperatura e duração da iluminação diária e fatores intrínsecos como reprodução e atividade preferencialmente noturna contribuem para maiores diversidades nos meses mais quentes do ano e na interface madrugada/amanhecer.

\section{Agradecimentos}

Os autores agradecem ao Prof. Dr. Everton Rodolfo Behr (UFSM) pelo auxílio na identificação dos exemplares e Rudi dos Santos, Sidinei dos Santos e Fabrício Veríssimo pela contribuição nas coletas. Bethânia Ross Copatti agradece pela bolsa do PIBIC-Unicruz.

\section{Referências Bibliográficas}

APONE, F., OLIVEIRA, A.K. \& GARAVELLO, J.C. 2008. Composição da ictiofauna do rio Quilombo, tributário do rio Mogi-Guaçu, Bacia do alto rio Paraná, sudeste do Brasil. Biota Neotrop. 8(1): www.scielo.br/pdf/ bn/v8n1/a12v8n1.pdf (último acesso em 06/06/2011).

AZEVEDO, C.L.O., PESSANO, E.F.C., TOMASSONI, D.S., QUERO, M.V.M. \& QUERO, E.C. 2003. Diversidade específica, densidade e biomassa da ictiofauna da nascente do arroio Felizardo, Bacia do rio Uruguai Médio, Uruguaiana, RS, Brasil. Biodiv. Pampeana 1(1):35-45. 
BEHR, E.R. 2005. Estrutura da comunidade e alimentação da ictiofauna dominante do rio Ibicui, RS. Tese de Doutorado, Pontifícia Universidade Católica do Rio Grande do Sul, Porto Alegre.

BERTOLETTI, J.J., LUCENA, C.A.S., LUCENA, Z.M.S., MALABARBA, L.R. \& REIS, R.E. 1989a. Ictiofauna do rio Uruguai superior entre os municípios de Aratiba e Esmeralda, Rio Grande do Sul, Brasil. Comun. Mus. Cienc. Tecnol. PUCRS, Ser. zool. 32(48):3-42.

BERTOLETTI, J.J., LUCENA, C.A.S., LUCENA, Z.M.S., MALABARBA, L.R. \& REIS, R.E. 1989b. Ictiofauna do rio Canoas, sistema do rio Uruguai Superior, Campos Novos, Santa Catarina, Brasil. Comun. Mus. Cienc. Tecnol. PUCRS, Ser. zool. 32(49):43-75.

BERTOLETTI, J.J., LUCENA, C.A.S., LUCENA, Z.M.S., MALABARBA, L.R. \& REIS, R.E. 1990. Estrutura e composição da fauna de peixes na área de influência da futura Usina Hidrelétrica de Garabi, Rio Grande do Sul, Brasil. Comun. Mus. Cienc. Tecnol. PUCRS, Ser. zool. 3(2):33-97.

BOSSEMEYER, I.M.K., WEIS, M.L.C., BENEMANN, S.T. \& BIER, M.L.S. 1985. Ictiofauna do Rio Santa Maria, RS. Cienc. nat. 7:209-222.

BRAGA, F.M.S. 2004. Hábitat, distribuição e aspectos adaptativos de peixes da microbacia do Ribeirão Grande, Estado de São Paulo, Brasil. Acta Sci. 26:31-36.

BUCKUP, P.A., MENEZES, N.A. \& GHAZZI, M.S. 2007. Catálago das espécies de peixes de água doce do Brasil. Museu Nacional, Rio de Janeiro, p. 11-14.

BUCKUP, P.A.\& REIS, R.E. 1997. Characidiin Genus Characidium (Teleostei, Characiformes) in Southern Brazil, with Description of Three New Species. Copeia 3:531-548. http://dx.doi.org/10.2307/1447557

CASATTI, L. \& CASTRO, R.M.C. 1998. A fish community of the São Francisco River headwaters riffles, southeastern Brazil. Ichthyol. explor. freshw. 9(3):229-242.

CASATTI, L., CASTRO, R.M.C. \& LANGEANI, F. 2001. Peixes de riacho do Parque Estadual Morro do Diabo, Bacia do alto rio Paraná Peixes de riacho do Parque Estadual Morro do Diabo, Bacia do alto rio Paraná, SP. Biota Neotropica1(1):1-15.

CASTRO, R.J., FORESTI, F. \& CARVALHO, E.D. 2003a. Composição e abundância da ictiofauna na zona litorânea de um tributário, na zona de sua desembocadura no reservatório de Jurumirim, Estado de São Paulo, Brasil. Acta sci., Biol. sci. 25(1):63-70.

CASTRO, R.M.C., CASATTI, L., SANTOS, H.F., FERREIRA, K.M., RIBEIRO, A.C., BENINI, R.C., DARDIS, G.Z.P., STOPIGLIA, R., ABREU, T.X., BOCKMANN, F.A., CARVALHO, M., GIBRAN, F.Z. \& LIMA, F.C.T. 2003b. Estrutura e composição da ictiofauna de riachos do Rio Paranapanema, Sudeste e Sul do Brasil. Biota Neotrop.3(1): www. biotaneotropica.org.br/v3n1/pt/fullpaper?bn01703012003_1+pt (último acesso em 07/06/2011)

CLESCERI, L.S.\& GREENBERG, A.E. 2005.Standard methodos for examination of water wastewater.21nd ed. American Public Health Association, Washington, 1600p.

COPATTI, C.E., ZANINI, L.G., VALENTE, A. 2009. Ictiofauna da microbacia do rio Jaguari - Jaguari/RS. Biota Neotrop. 9(2): www.biotaneotropica.org.br/ v9n2/pt/abstract?inventory+bn00809022009 (último acesso em 07/06/2011).

DALA-CORTE, R.B., FRANZ, I., BARROS, M.P. \& OTT, P.H. 2009 Levantamento da ictiofauna da Floresta Nacional de Canela, na região superior da Bacia hidrográfica do Rio Caí, Rio Grande do Sul, Brasil. Biota Neotrop. 9(2): www.scielo.br/pdf/bn/v9n2/a21v09n2.pdf (último acesso em 07/06/2011).

JACKSON, D.A., PERES-NETO, P.R. \& OLDEN, J.D. 2001. What controls who is where in freshwater fish communities - the roles of biotic, abiotic and spatial factors. Can. J. Fish. Aquat. Sci. 58:157-170. http://dx.doi. org/10.1139/cjfas-58-1-157

JUNGWIRTH, M., MUHAR, S. \& SCHMUTZ, S. 2000. Fundamentals of fish ecological integrity and their relation to the extended serial discontinuity concept. Hydrobiologia 422-423:85-97. http://dx.doi. org/10.1023/A:1017045527233
KAUFMANN, V. \& PINHEIRO, A. 2009. Relações entre diversidade íctia e fatores hidrodinâmicos de um riacho na Bacia do Rio Uruguai. Biota Neotrop. 9(1):www.biotaneotropica.org.br/v9n1/pt/ abstract?article+bn00809012009 (último acesso em 06/06/2011).

LOWE-McCONNELL, R.H. 1975. Fish Communiteis in Tropical Freshwaters. Longman, New York, $337 \mathrm{p}$.

MALABARBA, L.R. \& ISAIA, E.A. 1992. The fresh water fish fauna of the rio Tramandaí drainage, Rio Grande do Sul, Brazil, with a discussion of its historical origin. Comun. Mus. Cienc. PUCRS, Ser. zool. 5(12):197-223.

MALABARBA, L.R. \& REIS, R.E. 1987. Manual de técnicas para a preparação de coleções zoológicas. 36. Peixes. Sociedade Brasileira de Zoologia, Curitiba, p. 1-14.

McAlEECE, N., LAMBSHEAD, P.J.D., PATERSON, G.L.J. \& GAGE, J.G. 1997.Biodiversity professional.The Natural History Museum and the Scottish Association for Marine Sciences, London, Beta-Version.

NELSON, J.S. 1994. Fishes of the world. 13nd ed. John Wiley \& Sons, New York, $600 \mathrm{p}$.

OKADA, E.K.A., AGOSTINHO, A. A., PETRERE JUNIOR, M. \& PENCZAK, T. 2003. Factors affecting fish diversity and abundance in drying ponds and lagoons in the upper Paraná River basin, Brazil. Ecohydrol. Hidrobiol. 3(1):97-110.

OLIVEIRA, A.K. \& GARAVELLO, J.C. 2003. Fish assemblage composition in a tributary of the Mogi Guaçu river basin, southeastern Brazil. Iheringia, Zool. 93(2):127-138. http://dx.doi.org/10.1590/S0073-47212003000200002

PERES-NETO, P.R., BIZERRIL, C.R.S.F. \& IGLESIAS, R.1995. An overview of some aspects of river ecology: a case study on fish assemblages distribution in an eastern Brazilian coastal river. In Estrutura, funcionamento e manejo de ecossistemas brasileiros (F.A. Esteves). Universidade Federal do Rio de Janeiro, Rio de Janeiro, p. 317-334. (Série Oecologia Brasiliensis).

PESSANO, E.F.C., AZEVEDO, C.L.O., QUEROL, M.C.M., QUEROL, E., BRASIL, L.G., CASTRO, L.R.B., PINTO, T.B. \& CORREAA, F.V. 2005. Ictiofauna do arroio Quarai-Chico, Bacia do médio rio Uruguai, no interior do Parque Estadual do Espinilho, Rio Grande do Sul, Brasil. Biotemas 18(2):143-153.

PIANKA, E.R. 1974. Evolutionary ecology. Harper \& Row, New York, 356 p.

REIS, R.E., WEBER, C. \& MALABARBA, L.R. 1990. Review of the Hypostomus Lacépède, 1803 from Southern Brazil, with descriptions of three new species (Pisces, Siluriformes, Loricariidae). Rev. Suisse Zool. 97(3):729-766.

ROSSO, J.J. 2006. Peces Pampeanos guia y Ecologia. L.O.L.A, Buenos Aires, $221 \mathrm{p}$.

SACCOL-PEREIRA, A. \& FIALHO, C.B. 2010. Seasonal and diel variation in the fish assemblage of a Neotropical delta in southern Brazil. Iheringia, Zool. 100(2):169-178. http://dx.doi.org/10.1590/S007347212010000200013

SHIBATTA, O.A., GEALH, A.M. \& BENNEMANN, S.T. 2007. Ictiofauna dos trechos alto e médio da Bacia do rio Tibagi, Paraná, Brasil. Biota Neotrop. 7(1): www.scielo.br/pdf/bn/v7n2/a14v07n2.pdf (último acesso em 07/06/2011).

VIANA, J.P. 1989. Estrutura da comunidade dos peixes do Ribeirão Sant'ana (Brasília, DF) ao longo de gradientes ambientais. Dissertação de Mestrado, Universidade de Brasília, Brasília.

WAITE, I.R. \& CARPENTER, K.D. 2000.Associations among fish assemblage structure and environmental variables in Willamette basin streams, Oregon. Trans. Am. Fish. Soc. 129:754-770. http://dx.doi.org/10.1577/15488659(2000)129\%3C0754:AAFASA\%3E2.3.CO;2

WEIS, M.L.C., BOSSEMEYER, I.M.K., BIER, M.L.S. \& LIPPOLD, H.O. 1983. Inventário da fauna ictiológica do rio Ibicui-Mirim, RS. Cienc. nat. 5:135-152.

WOOTTON, R.J. 1990. Ecology of Teleost Fishes. Chapman \& Hall, London, 404 $\mathrm{p}$.

YOSSA-PERDOMO, M.I. 2002. Estratégia alimentar de peixes detritívoros das Bacias do Orinoco e do Amazonas. Tese de Doutorado, Universidade do Amazonas, Manaus.

ZANIBONI FILHO, E., MEURER, S., SHIBATTA, O.A. \& NUÑER, A.P.O. 2004. Catálogo ilustrado de peixes do alto rio Uruguai. Universidade Federal de Santa Catarina, Florianópolis, 128 p. 
